\title{
An Optimized Flight Control System of Quad-rotor Aircraft
}

\author{
Xiaoning Chen ${ }^{1, a}$, Hongting Dong ${ }^{1, b}$, Dexiang Zhang ${ }^{1, c}$, Jing Xia ${ }^{1, d}$ \\ ${ }^{1}$ College of Electrical Enginerring and Automation, Anhui university, \\ Anhui 230601, China;

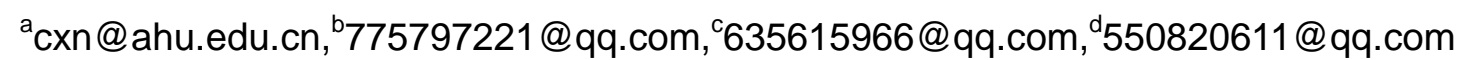

Keywords: optimization, csyncretic data of gyroscope, accelerometer and magnetometer , fuzzy algorithm , joint simulation

\begin{abstract}
The flight performance of four-rotor unmanned helicopter depends largely on the flight control system, the flight control system was mainly consisted of attitude algorithm and controller. During design time, firstly, in order to real-time update for the quaternion , we fused the data of gyroscope accelerometer and magnetometer. And we obtained the attitude angle through quaternion to Euler angle conversion. Then, we calculated the output of throttle by fuzzy algorithm, which was used to control the attitude. And control algorithm was used and optimized by the method of the joint simulation. Finally, four-rotor unmanned helicopter was connected to PC with bluetooth and we obtained the air data of UAV form PC. The data display that the fuzzy controller can keep the stability of flying.
\end{abstract}

\section{Introduction}

Multi-rotors aircraft gained more and more attention from researchers because of its characteristics, such as vertical take-off and landing in a complex environment, hovoring ,easy to navigate and so on ${ }^{[1]}$. Also aircrafts have a widely application in aerial photo ,security and mapping fields.

X-mode multi-rotor aircraft is a kind of relative simple model aircraft. They are cheap, convenient to carry, easy to maintain. X-mode multi-rotor aircraft has six degrees of freedom. They can motion like roll, pitching yaw by the change of rotating speed of two pairs of motor when flying.

To make the aircraft maintain a well and stable flight attitude in the air and response to control fast ,this paper adopts more accurate angle calculating methods and optimized control algorithms aiming at designing a flying control system carried on X-mode four-rotor aircraft .And then it verified that the flying control system can control air vehicle stably and affectively via upper computer results.

\section{Attitude Computation}

\subsection{Angle Integration}

Air vehicle posture computation is the basic of the whole flight control system, which usually referred to as "foundation" .The drift caused by gyroscope long working time need adjust .For this normal solutions is mixing accelerometer with reference quantity to revise angular speed, calculating quaternion ,to conducted angle verification, namely "six-axis integration". The method is relatively simple, angle change in yaw direction can go away when after long flying or a greater attitude change. This can generate spin, which is bad for stable flight.

The characteristic which gyroscope short time measurement is accurate, accelerometer long time test is stable, magnetometer horizontal azimuth measurement is precise is important .This paper integrates gyroscope ,accelerometer, magnetometer together using merged data ,real time updating quaternion to get to aircraft attitude computation and update. The method has a higher precision and avoid drift in yaw direction. The comparison between two methods' results is shown in figure 1. 


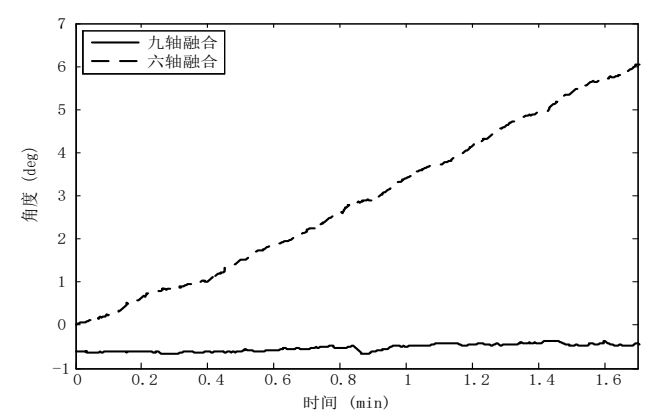

Fig. 1 Comparison to two computation methods

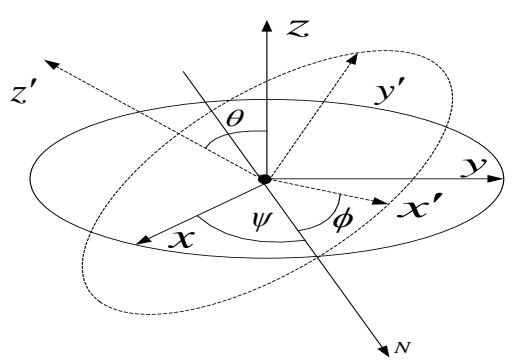

Fig. 2 Euler angles representations

We can learn from the above picture that there existed drift in yaw direction seriously on the condition only integrating gyroscope and accelerometer .However, nine-axis integration adding magnetometer is essentially unchanged when static. Thus we can draw the conclusion that the angle attitude optimized yaw error.

\subsection{Quaternion Computation}

Quaternion can described as a kind of coordinate system or a vector rotation relative to a coordinate system. When one any direction coordinate system $\mathrm{B}$ rotate $\theta$ angle relative to another any direction coordinate system A,they can express as (1):

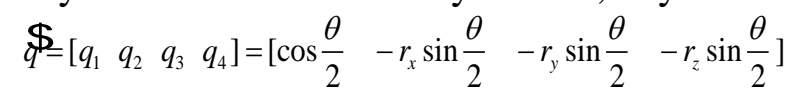

$r_{x}, r_{y}, r_{z}$ respectively represents $x$-axis,y-axis,z-axis unit vector in coordinate system.q $\wedge^{\wedge}$ can be described as rotation matrix shown as follow:

$$
R=\left(\begin{array}{ccc}
2 q_{1}^{2}-1+2 q_{2}^{2} & 2\left(q_{2} q_{3}+q_{1} q_{4}\right) & 2\left(q_{2} q_{4}-q_{1} q_{3}\right) \\
2\left(q_{2} q_{3}-q_{1} q_{4}\right) & 2 q_{1}^{2}-1+2 q_{3}^{2} & 2\left(q_{3} q_{4}+q_{1} q_{2}\right) \\
2\left(q_{2} q_{4}+q_{1} q_{3}\right) & 2\left(q_{3} q_{4}-q_{1} q_{2}\right) & 2 q_{1}^{2}-1+2 q_{4}^{2}
\end{array}\right)
$$

The rotation of air vehicle in space can express as Euler Angles $\psi, \theta, \phi$, which represents three attitude angle .Attitude angle showed as quaternion are (3)-(5):

$$
\begin{aligned}
& \psi=A \tan 2\left(2 q_{2} q_{3}-2 q_{1} q_{4}, 2 q_{1}^{2}+2 q_{2}^{2}-1\right) \\
& \theta=-\sin ^{-1}\left(2 q_{2} q_{4}+2 q_{1} q_{3}\right) \\
& \phi=A \tan 2\left(2 q_{3} q_{4}-2 q_{1} q_{2}, 2 q_{1}^{2}+2 q_{4}^{2}-1\right)
\end{aligned}
$$

\section{Attitude Control}

Attitude control is the main aspect of the flight system mentioned in this paper. Simulation contrast is necessary when choosing control method. Traditional PID is unable to realize accurate control due to X-mode four-rotor aircraft strong nonlinear, multi variable coupling, under actuation ${ }^{[2]}$. Cascade stage PID is used in simulation to replace traditional ones, compared with fuzzy control algorithm and thus come to the conclusion that robustness of fuzzy control algorithm is much better.

\subsection{Simulation Model Building}

In the process of mathematic model building, this paper adopts intuition union simulation. This paper designs three-dimensional structure model graph using Unigraphics NX according to four-rotor aircraft airframe in figure 3. And then imports them to dynamics analysis software Adams with the format .X_T ( parasolid ).At the same time ,X-mode four-rotor aircraft are built when every components and inertia of the model are defined to make them have physic characteristics of real prototype .With this , union simulation based on Unigraphics ,Adams ,and Matlab are finished.

\subsection{Fuzzy Control Design}

Fuzzy control is adding a fuzzy control rule into traditional PID control to enhance robustness and adaptivity of whole control system ${ }^{[3][4]}$. Control rule of fuzzy PID controller is as (6): 
$u(t)=k_{F p} e(t)+k_{F i} \int_{0}^{t} e(t) d t+k_{F d} \frac{d}{d t} e(t)$

$k_{F p}=F_{\text {fuzyy }}\left(e(t), \frac{d}{d t} e(t), \int_{0}^{t} e(t)\right) \quad k_{F i}=F_{\text {fuzyy }}\left(e(t), \frac{d}{d t} e(t), \int_{0}^{t} e(t)\right) k_{F d}=F_{f u z z y 3}\left(e(t), \frac{d}{d t} e(t), \int_{0}^{t} e(t)\right)$

$F_{\text {fuzzyn }}()$ was fuzzy function about $e(t), \frac{d}{d t} e(t), \int_{0}^{t} e(t) n=1,2,3$.

Control system is shown as figure 4 ,input of fuzzy control is variable error, namely deviation between desire angle and current angle. This paper increases angular speed as the second input aiming at accelerating system response speed. Meanwhile, optimized parameters, which are dynamic, can be gained according to fuzzy algorithm system different kinds of control condition. Compared with static parameter of traditional PID controller, Fuzzy PID controller dynamic parameters adaptation is stronger when system surroundings changed.

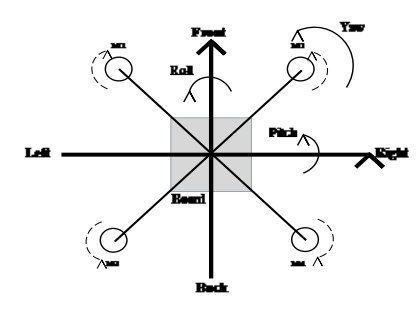

Fig. 3 Airframe model of four-rotor aircraft

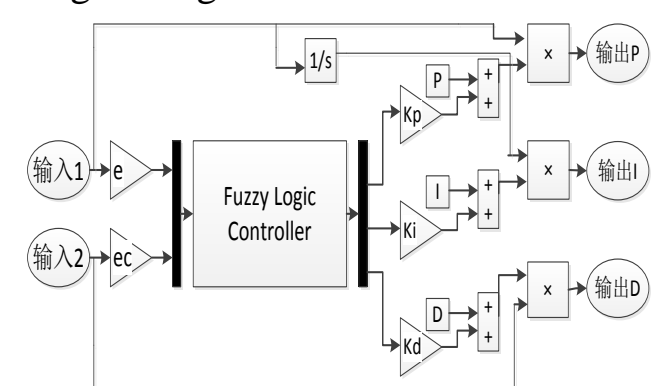

Fig. 4 Fuzzy controller inner structure

\subsection{Simulation Results Comparison}

After building X-mode four-rotor aircraft union simulation system, two different control methods results can be observed once desire signal is set up to simulate. In the process of simulation, there exists two sets of test, one is the worst signal, which step signal is expectation with value 10(desire angle is 10 degree),the other one is at the equal condition except for adding random numerical value as interference. Under this circumstances, compared graph is shown as follow:

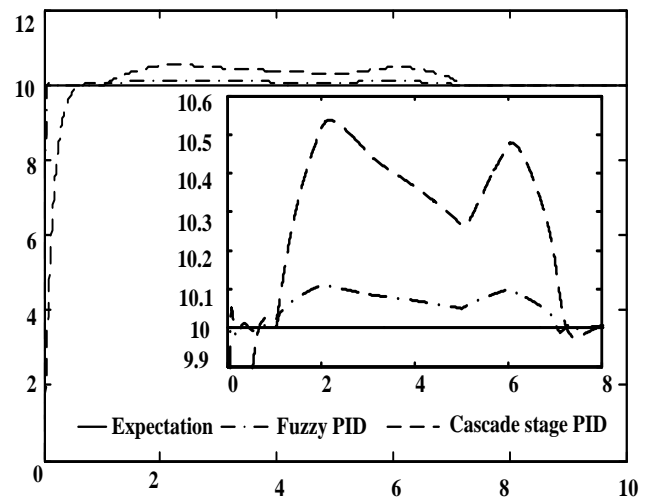

Fig. 5 Comparison in pitching direction

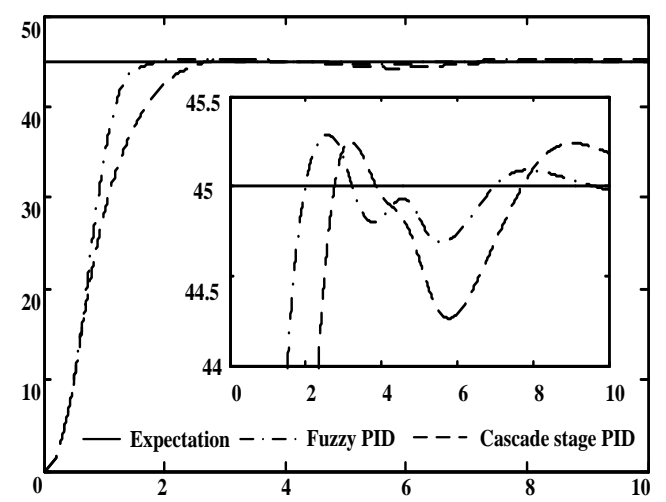

Fig. 6 Comparison in yaw direction

$\mathrm{X}$-mode four-rotor unmanned aerial vehicle airframe has a high symmetry, so chart between pitching direction and roll direction is nearly the same .Here pitching direction for example, response comparison is shown as chart 1.

Chart 1 Two control methods Comparison

\begin{tabular}{|c|c|c|c|c|}
\hline \multirow[t]{2}{*}{ Item } & \multicolumn{2}{|c|}{$\begin{array}{c}\text { Comparison in Pitching } \\
\text { Direction }\end{array}$} & \multicolumn{2}{|c|}{$\begin{array}{c}\text { Comparison in Yaw } \\
\text { Direction }\end{array}$} \\
\hline & Cascade Stage & Fuzzy & Cascade Stage & Fuzzy \\
\hline Response Time & $0.1 \mathrm{~s}$ & $0.02 \mathrm{~s}$ & $1 \mathrm{~s}$ & $0.8 \mathrm{~s}$ \\
\hline Overshoot & $0.28 \%$ & $0.50 \%$ & $1 \%$ & $0.20 \%$ \\
\hline Regulation Time & $0.7 \mathrm{~s}$ & $0.07 \mathrm{~s}$ & $2.9 \mathrm{~s}$ & $2 \mathrm{~s}$ \\
\hline Peak Time & $0.87 \mathrm{~s}$ & $0.095 \mathrm{~s}$ & $4 \mathrm{~s}$ & $2.8 \mathrm{~s}$ \\
\hline
\end{tabular}

From the above chart we can know that fuzzy controller has a faster response speed, a smaller 
overshoot, a higher control precision, a stronger capacity of resisting disturbance ,a greater control effect than cascade controller.

\section{Simulation Result Verification}

The paper set up practical X-mode four-rotor aircraft for verifying system described above real performance. The aircraft adopts STM32F407 as main controller, programmed with C language, linked MPU9150 with HMC5883 to realize attitude calculation by I2C port. At the same time , the aircraft provides power for system using brushless electric machine and electron speed regulator; when in the process of flight, remote control can command airframe and uploading data to upper computer. The results are shown as follows:

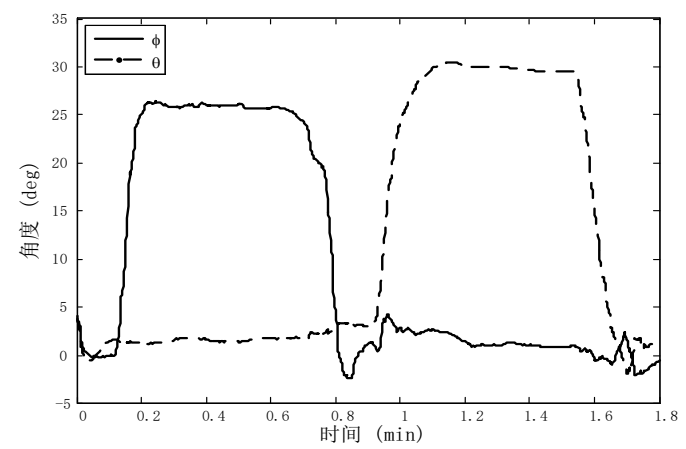

Fig. 7 Tracking result of flying attitude angle

From the picture above we can easily make out that aircraft with the flight control system has better robustness and reaction speed, faster angle tracing.

\section{Ending}

$\mathrm{X}$-mode four-rotor unmanned aircraft is a kind of common air vehicle. Flight control system is the key element whether the aircraft can fly stable or not .This paper uses nine-axis integration angle calculation and fuzzy algorithm control method to optimize control system. What is more, this paper applies the system mentioned in the article to X-mode four-rotor, the aircraft flies stable and finish various of movement such as forward, left shift, dextroversion and so on.

\section{Acknowledgements}

Throughout this work we would like to express our particular gratitude to our colleagues for helpful conversions and inspiration in the course of this research. The support from the Chinese National Nature Science Foundation Grant(61272025) for this research are gratefully acknowledged as well.Also corresponding author Hongting Dong helps a lot in the paper.

\section{References}

[1] Jilong Yue,Qingjie Zhang,Huayong Zhu.Brief talk on microminiature four-rotor unmanned aircraft research process and key technology.Electronics Optics \&Control,2010,10(10):46-52.

[2] Chunhui Zhang.Research on high accuracy algorithm of strapdown inertial navigation system.Harbin Engineering University,2005.

[3] Lu Wang.Research on control technique based on four-rotor unmanned aircraft.Harbin Engineering University,2012.

[4] Tao Wang,Huiming Zhang.Union control system simulation based on ADAMS and MATLAB.Mechanical Engineering \& Automation ,2005(3):79-81. 